\title{
A proposed approach to accelerate evidence generation for genomic-based technologies in the context of a learning health system
}

\author{
Christine Y. Lu, MSc, PhD ${ }^{1}$, Marc S. Williams, MD², Geoffrey S. Ginsburg, MD, PhD ${ }^{3}$, \\ Sengwee Toh, $\mathrm{ScD}^{1}$, Jeff S. Brown, $\mathrm{PhD}^{1}$ and Muin J. Khoury, MD, PhD ${ }^{4}$
}

Genomic technologies should demonstrate analytical and clinical validity and clinical utility prior to wider adoption in clinical practice. However, the question of clinical utility remains unanswered for many genomic technologies. In this paper, we propose three building blocks for rapid generation of evidence on clinical utility of promising genomic technologies that underpin clinical and policy decisions. We define promising genomic tests as those that have proven analytical and clinical validity. First, risksharing agreements could be implemented between payers and manufacturers to enable temporary coverage that would help incorporate promising technologies into routine clinical care. Second, existing data networks, such as the Sentinel Initiative and the National Patient-Centered Clinical Research Network (PCORnet) could be leveraged, augmented with genomic information to track the use of genomic technologies and monitor clinical outcomes in millions of people. Third, endorsement and engagement from key stakeholders will be needed to establish this collaborative model for rapid evidence generation; all stakeholders will benefit from better information regarding the clinical utility of these technologies. This collaborative model can create a multipurpose and reusable national resource that generates knowledge from data gathered as part of routine care to drive evidence-based clinical practice and health system changes.

Genet Med advance online publication 10 August 2017

Key Words: Big Data; clinical utility; data networks; evidence; genomics

\section{INTRODUCTION}

Since the completion of the Human Genome Project more than a decade ago, the fields of genome sciences and to a lesser degree genomic medicine have advanced rapidly. Nextgeneration sequencing technologies have substantially improved the accuracy and reduced turnaround times and costs, enabling their use as clinical testing platforms for whole-exome sequencing, whole-genome sequencing, and next-generation gene panels. These technological advancements have spurred interest in personalized and precision medicine. The Centers for Medicare and Medicaid Services and the US Food and Drug Administration (FDA) have important roles in the regulation of genomic-based technologies in the United States. Through the Clinical Laboratory Improvement Amendments, the Centers for Medicare and Medicaid Services require tests to demonstrate analytical validity (i.e., a test's ability to accurately and reliably measure the genotype of interest) but it does not require demonstration of clinical validity (i.e., a test's ability to detect or predict the associated disorder (phenotype)). ${ }^{1}$ The FDA currently only regulates tests sold as kits although it has proposed to oversee tests that detect medically important genomic variations. ${ }^{2,3}$ There is a growing body of scientific evidence to support the clinical validity of genomic tests, and the FDA supports greater use of publicly accessible genetic variant databases. ${ }^{3}$ For instance, the ClinVar database ${ }^{4}$ accessible through the ClinGen Resource is an interconnected community resource to improve our understanding of genomic variation and improve use of genomic information in clinical care.

Importantly, however, evidence of clinical utility remains elusive for the majority of genetic and other omics-based tests, with the exception of companion diagnostics, which are often codeveloped with drugs. ${ }^{5}$ Clinical utility of a test refers to the ability of a test's result to improve measurable clinical outcomes and add value to patient-management decision making. ${ }^{6,7}$ Evidence of clinical utility might include demonstrated benefits and harms associated with a test, improvements in patient care, effects on patient outcomes, and effects on patient or family member decision making. ${ }^{7}$ Evidence of clinical utility is an important piece of information for both insurance coverage and clinical adoption. Insurance coverage decisions and treatment decisions are complex. Apart from scientific evidence, other value judgments also come into play (e.g., whether services are deemed "medically necessary") but how these influence coverage decisions differs across

${ }^{1}$ Department of Population Medicine, Harvard Medical School and Harvard Pilgrim Health Care Institute, Boston, Massachusetts, USA; ${ }^{2}$ Genomic Medicine Institute Geisinger Health System, Danville, Pennsylvania, USA; ${ }^{3}$ Center for Applied Genomics \& Precision Medicine, Duke University, Durham, North Carolina, USA; ${ }^{4}$ Office of Public Health Genomics, Centers for Disease Control and Prevention, Atlanta, Georgia, USA. Correspondence: Christine Y. Lu (christine_lu@harvardpilgrim.org) 
payers. Economic evaluations also depend upon clinical utility evidence. ${ }^{8}$ Similarly, treatment decisions are complex, and information from genomic tests is considered along with the patient's history, clinical status, diagnosis, treatment options, and so on. Despite the complexity of decisions, generating clinical utility evidence would benefit many. Efforts are ongoing to develop this evidence but a randomized controlled trial for every new stand-alone genomic-based technology is not feasible given the number of tests that have been developed, costs, and small patient populations due to the nature of individualized medicine. The paradigm where a test is developed for a single indication is outdated. ${ }^{9,10}$ Once a genome or exome is obtained for any purpose, the information from the sequence can be used for many purposes across the lifetime of the patient, making the scope and time frame of a single clinical trial untenable.

In this paper, we propose three building blocks for rapid generation of clinical utility evidence for "promising" genomic technologies. We define promising genomic tests below. The first of the three building blocks for rapid generation of clinical utility evidence is risk-sharing agreements between payers and manufacturers that could enable temporary coverage, which would help incorporate promising technologies into routine clinical care. Manufacturers in this model could include manufacturers of the technology (e.g., Illumina), diagnostic companies affiliated with large pharmaceutical companies (e.g., Roche Molecular Systems), companies focused exclusively on genetic and genomic testing (e.g., Myriad Genetic Laboratories, Genomic Health), and large reference laboratories (e.g., Quest Diagnostics). The second is leveraging existing data networks, which need to be augmented with genomic information to track the use of genomic technologies and monitor clinical outcomes in millions of people. Third, endorsement and engagement from key stakeholders will be needed to establish this collaborative model for rapid evidence generation; all stakeholders will benefit from better information regarding the clinical utility of these technologies. We discuss these building blocks below and present a summary in Table 1. "Promising" technologies are those that have proven analytical and clinical validity. While they have early evidence of impact on clinical care, the available evidence is insufficient to support widespread adoption in clinical practice. In other words, promising technologies are tier 2 tests based on the three-tier classification system created by the Office of Public Health Genomics at the US Centers for Disease Control and Prevention; this classification system sorts genomic tests by level of evidence. ${ }^{11}$ Currently, there are 105 tests under the tier 2 category. This is likely an underestimate as rapidly developing genomic data will require more frequent updates by the research community.

\section{RISK-SHARING ARRANGEMENTS FOR RAPID EVIDENCE GENERATION}

Typically by covering a technology, payers (and their enrollees) bear the financial risk of the purchase if the technology does not result in improved outcomes commensurate with its cost, or worse if outcomes are unchanged or worsen. Risk-sharing agreements (RSAs), also known as managed entry agreements, are implemented to overcome the challenges of financial risks associated with uncertain clinical effectiveness, cost-effectiveness, and/or budget impact and at the same time enable timely, subsidized patient access to technologies that would otherwise not be covered. ${ }^{12,13}$ These include financial-based or outcomes-based agreements between payers and manufacturers to spread the associated financial risks and uncertainties, or coverage with evidence development schemes to collect more data through research. ${ }^{14,15}$ RSAs are typically established for high-cost technologies (e.g., biologics, positron emission tomography imaging) because the financial consequences of any uncertainties are greater in magnitude. In the United States, most RSAs are coverage with evidence development $(\mathrm{CED})^{16}$ programs through the Centers for Medicare and Medicaid Services. The CED program links coverage of a health technology to a requirement that patients participate in a registry or clinical trial. Of the 22 CEDs to date only one pharmacogenetic test has been approved (the CED for testing with use of the anticoagulant drug warfarin was active from 2009 through 2014). ${ }^{17}$

For promising genomic technologies we propose temporary coverage coupled with RSAs while clinical utility evidence is being accumulated. The expense of demonstrating the test's analytical and clinical validity would remain with the manufacturer, but payers and manufacturers share the financial risk of coverage of promising technologies that may or may not prove to have clinical utility. Payers and manufacturers should predefine and agree on what constitutes "sufficient" clinical utility and the timeline for making the evaluation for each individual test. Coverage would be withdrawn when subsequent evidence shows tests do not provide sufficient clinical utility or expanded if evidence suggests sufficient clinical utility. Coverage would enable patient access to these technologies as part of routine care while withdrawal of coverage could lead to substitution, that is, use of alternative tests instead. Longitudinal data on use of genomic technologies and subsequent treatments could be routinely captured by administrative and electronic health record (EHR) databases. Multiple health plans and healthcare organizations participating in an RSA for a single genomic technology would allow rapid generation of evidence for that technology among larger populations. This model would be preferred over registries or clinical trials as in CED programs because recruitment and data generation are known to be slow and the need to rigorously define inclusion and exclusion criteria may limit the applicability to real-world situations. ${ }^{18}$ Payers and manufacturers can share the financial burden of evidence generation for genomic technologies through a nonprofit, scientific public service initiative that together conducts research and pays for evidence generation and even new data collection. The Biologics and Biosimilars Collective Intelligence Consortium is an existing, collaborative 
Table 1 Rapid evidence generation for genomic technologies: current and proposed paradigms

\begin{tabular}{|c|c|c|c|}
\hline & & Current paradigm & Proposed paradigm \\
\hline \multicolumn{2}{|l|}{ Evidence of clinical utility } & $\begin{array}{l}\text { Absent for many genomic technologies as } \\
\text { randomized controlled trials are not an } \\
\text { economically feasible design in this context }\end{array}$ & $\begin{array}{l}\text { Evidence generation is possible through three building } \\
\text { blocks for a collaborative model: (i) risk sharing } \\
\text { between payers and manufacturers to enable } \\
\text { temporary coverage of promising genomic tests, (ii) } \\
\text { leveraging existing data networks with necessary } \\
\text { advances for integrating genomic information, and (iii) } \\
\text { endorsement and engagement from key stakeholders }\end{array}$ \\
\hline \multicolumn{2}{|l|}{ Insurance coverage } & $\begin{array}{l}\text { No market access or low utilization of many } \\
\text { genomic technologies for manufacturers }\end{array}$ & Risk sharing between payers and manufacturers \\
\hline \multicolumn{2}{|l|}{$\begin{array}{l}\text { Efforts to generate evidence } \\
\text { on clinical utility }\end{array}$} & Disease-, study-specific efforts & $\begin{array}{l}\text { Accruing real-time data among large populations } \\
\text { within a single, large health-data network }\end{array}$ \\
\hline \multirow[t]{3}{*}{$\begin{array}{l}\text { Data elements necessary } \\
\text { for determining clinical } \\
\text { utility }\end{array}$} & $\begin{array}{l}\text { Patient } \\
\text { demographics, } \\
\text { identifiers }\end{array}$ & Present in EHR data if used & Present in insurance claims and EHR data \\
\hline & $\begin{array}{l}\text { Genomic test } \\
\text { order, utilization, } \\
\text { results }\end{array}$ & $\begin{array}{l}\text { Poorly captured in some EHR systems if } \\
\text { used }\end{array}$ & $\begin{array}{l}\text { Advances are needed to (i) better capture tests } \\
\text { performed including specific billing codes, (ii) make test } \\
\text { results easily accessible in electronic health data, (iii) } \\
\text { build a common data model to aggregate data from } \\
\text { different health systems and insurers }\end{array}$ \\
\hline & $\begin{array}{l}\text { Subsequent } \\
\text { treatments/ } \\
\text { management }\end{array}$ & Incomplete records in EHR systems if used & $\begin{array}{l}\text { Insurance claims and EHR data contain fairly complete } \\
\text { medical and pharmacy utilization records }\end{array}$ \\
\hline
\end{tabular}

EHR, electronic health records; NIH, National Institutes of Health.

model of this kind. ${ }^{19}$ The proposed model is best suited to integrated delivery systems (e.g., Kaiser and Veterans Affairs systems), but could work with fully insured plans and other models. This is because of the complexity of public and private payers. Particularly private payers are in many cases simply administering insurance services as decided by employers that self-insure. Although, the evidence generated could also benefit people with self-insured plans.

Potential limitations include payers' and manufacturers' interest in entering into an RSA for a genomic technology. For manufacturers, they could gain market access for promising technologies that would otherwise not be covered. For payers, although many genomic technologies are relatively inexpensive when compared to many drugs, genomic testing may be cost-saving if the results identify which patients are likely to benefit from the use of expensive pharmaceuticals. For instance, KRAS testing prior to the use of irinotecan for colorectal cancer, where only $15 \%$ of patients have a result from testing that confers effectiveness, would significantly reduce the health plans' reimbursement for this expensive medication. ${ }^{20} \mathrm{~A}$ potential barrier to the rapid generation of evidence is the participation of multiple parties in the RSAs. There might be a disincentive for multiple payers to participate in an agreement for a particular technology. For example, in our proposed model, if one payer is willing to cover a test in order to enable evidence generation and that information is made publicly available rapidly, it may be a disincentive for other payers to participate. This could limit the number and diversity of individuals receiving the technology, and potentially slowing down the generation of evidence.

\section{LEVERAGING EXISTING HEALTH-DATA NETWORKS FOR RAPID EVIDENCE GENERATION}

If promising technologies are covered by health plans, electronic health-care data related to their use, and subsequent use of health services, could accrue through routine care delivery and payment, ${ }^{21}$ creating a learning health-care system that generates knowledge for evidence-based clinical practice and health system changes. ${ }^{22,23}$ The approach is also 
in line with the new 21st Century Cures Act, which promotes innovation and real-world evidence. ${ }^{24}$ As discussed below, we believe administrative health-care databases contain many data components needed to assess clinical utility of genomic technologies. Thus, we could leverage existing data networks supplemented with genomic information for this purpose. We discuss this building block of our model below after a brief overview of other approaches that have been applied to gather evidence for genomic-based technologies.

First, consortium/multisite clinical trials have been established to evaluate clinical utility of specific tests, for example, CYP2C9/VKORC1 testing for warfarin treatment. ${ }^{25}$ Second, practice-based research networks of clinical practice groups, often driven by academic investigators, have contributed to the understanding of genetic and other biomarkers regarding treatment responses. ${ }^{26-28}$ Third, commercial laboratories have collaborated with care delivery systems to conduct studies assessing clinical validity and utility of new tests. ${ }^{29}$ Fourth, the Electronic Medical Records and Genomics (eMERGE) Network of nine distinct systems use electronic medical records as a tool for genomic research. ${ }^{30}$ While these approaches have worked to some extent, they have several limitations. These include limited scope (e.g., sample size, number of technologies that can be assessed, expertise of investigators), lack of longitudinal and comprehensive data capture (e.g., patients often seek care outside the practice-based networks), and length of time needed for recruitment and data collection.

Our model proposes the use of health-data networks that repurpose EHR and administrative data from large populations of patients ${ }^{31}$ to track the use of genomic technologies and monitor clinical outcomes. Such networks have already been established and are transforming health care, public health surveillance, and clinical research by providing timely real-world evidence of medical product safety and effectiveness. ${ }^{32,33}$ Health-data networks of multiple systems would leverage the investments of many organizations to provide a shared national Big Data resource that benefits patients, providers, payers, and manufacturers. The largest network is the FDA-sponsored Sentinel Initiative ${ }^{31}$ covering more than 380 million person-years of data; data are actively accruing on nearly 50 million people from 18 health plans. The data foundation of this program consists of insurance claims data supplemented by EHR data, laboratory test result data, and registry data. Another established network that would increase our understanding of clinical utility of genomic tests is the National Patient-Centered Clinical Research Network (PCORnet). ${ }^{31,34}$ This network, funded by the Patient-Centered Outcomes Research Institute (PCORI), involves electronic health-care data from 13 clinical data research networks and 20 patient-powered research networks. The breadth and depth of data derived from very large populations could allow fast generation of evidence on clinical orders of genetic testing, subsequent treatment and management of patients, and patient health outcomes-all of which are essential for understanding the clinical utility of genomic technologies.
The main barrier to using such data networks to assess clinical utility of genomic tests is that most health systems currently may not be able to capture sufficient information on the specific genetic test performed and the test results. The challenges can be overcome through stakeholder collaboration and partnerships. First, laboratories that perform tests typically store data elements on the specific tests and test results. Laboratories provide genomic test results back to the ordering clinician; thus, genomic information might exist as part of a patient's medical record in some EHR systems, although rarely as structured data. The main limitation to appropriate data can be overcome if these data can be integrated into EHR or other systems to enable efficient cross-institutional research. Efforts have already begun to add genomic information to existing data networks. For instance, the eMERGE network links EHR data to genomic information ${ }^{30}$ although only for a very small number of patients. For rapid evidence generation, additional challenges include limited incentives to build capacities integrating genomic information ${ }^{35}$ and patient privacy and data security concerns. Second, payers could require information on genomic test use and results as part of the reimbursement process for care, and thus could capture such information in claims data (e.g., if insurers target use of cetuximab for colorectal cancer to individuals with KRAS wild type, the treating physician would provide this information). A related challenge to overcome is the need for billing codes that are specific for a given genomic test. There is a transition to these types of codes from prior codes assigned to technical components of the test, rather than the test itself. Third, payers could augment their longitudinal administrative claims by contracting with laboratory service vendors to access results for enrollees that have tests completed by those vendors; adding information about tests performed and results would complete the longitudinal records of care of individual patients. ${ }^{36} \mathrm{~A}$ related challenge to overcome is that laboratories need to send genomic test results in a structured data format. ${ }^{37}$

Given the fragmented US health systems, no single system will have enough data to explain genotype-phenotype relationships, especially for rare diseases; therefore, data sharing across health systems is necessary. To accomplish this, common standards and protocols ${ }^{31}$ must be established to make sharing and computing data among many sites possible and efficient, which is the approach that underpins the successful health-data networks such as Sentinel and PCORnet. Consortiums such as eMERGE and Implementing Genomics in Practice (IGNITE) are actively developing a toolbox of measures for clinical utility evidence through the natural course of care; this initiative can help standardize such measures. ${ }^{38}$ Sentinel and PCORnet have also developed privacy-protecting data-sharing and analytic methods to overcome and minimize data privacy and security issues and the challenge of interoperability. ${ }^{39-41}$ Data from large numbers of patients through health-data networks can reflect the diversity of the underlying population such as race/ethnicity, age, and sex, and reduce biases and nongeneralizability. It is possible to design and perform prospective analyses of electronic health 
data as real-world experience of genomic technologies accrues in clinical practice. Prospective analyses can potentially produce actionable evidence more quickly than the conventional one-time retrospective analysis. ${ }^{42}$ The successes and lessons learned from Sentinel and PCORnet are helpful for the development of a model for generating clinical utility evidence. Our proposed approach of integrating or linking genomic information to existing health-data networks to enable rapid assessment of clinical utility of genomic tests is consistent with the Big Data movement, ${ }^{43}$ which aims to improve health by facilitating precision medicine, and enhancing disease prediction and prevention.

\section{STAKEHOLDER ENGAGEMENT FOR RAPID EVIDENCE GENERATION}

The success of our proposed model is contingent upon the collaboration between manufacturers, payers, and provider groups to share the financial risk, integrate (or link) genomic data into the current EHR and claims data systems, and support use of health-data networks. Uptake of promising genomic technologies by clinicians is an integral part of the model. To enable this, adequate clinical decision support (e.g., the eMERGE network and the IGNITE consortium ${ }^{44}$ ) and education are essential. Clearly, supports from EHR vendors, health-care systems, and government agencies are also needed. The business community that decides insurance benefits for private payers to administer is also an important stakeholder. In addition, patients, academics, and researchers are important partners in this collaborative model.

Patients have taken on an increasing interest and role in driving research agendas. ${ }^{45-48}$ Private and public groups (e.g., PCORI) have recently begun to focus on patient-centered research, thereby shifting the patient from the role of a research subject to a partner. ${ }^{49}$ Patients dealing with difficult clinical situations (e.g., a child with a complex, undiagnosed illness) may be particularly interested in promising but still unproven technologies. In our model, patients would have the option of selecting from proven genetic tests as well as promising tests that are covered temporarily. Several studies have reported high patient interest in genetic testing, including susceptibility testing, pharmacogenetic testing, and whole-genome or whole-exome sequencing. ${ }^{50-53}$ Therefore, we believe that patients would likely welcome and be active partners in our model of evidence generation for promising technologies. This has been realized in a single system, the MyCode Community Health Initiative at Geisinger Health System. ${ }^{54}$ We envision our model would be in line with the Privacy and Trust Principles under the Precision Medicine Initiative..$^{55}$ The model would encourage motivated patients to be engaged in generating research questions, contributing data, interpreting results, and communicating findings. By engaging patients as active collaborators, we could "allay their concerns about the potential for discrimination, thereby allowing individuals to take advantage of genetic testing, technologies, research, and new therapies," as stated by the Genomic Information Nondiscrimination Act of 2008. ${ }^{56}$
Finally, academics and researchers have an important role in the assessment of clinical utility of genomic technologies. Use of genomic testing and results and longitudinal records of health-care utilization to examine clinical utility requires large-scale epidemiologic studies and needs collaboration between multidisciplinary scientists and policy-makers including the fields of epidemiology, comparative effectiveness research, bioinformatics, clinical genetics, health policy, and public health. To assess cost-effectiveness following the demonstration of clinical utility requires collaboration with decision scientists and health economists. ${ }^{57}$ Data collected through our proposed model are observational in nature and therefore data analyses are vulnerable to many biases such as selection and confounding. Pragmatic trials may be an important alternative to observational studies. They have both high external and internal validity and costs can often be kept low because of the pragmatic design and data collection.

A related priority is capacity building to increase the number and quality of training of researchers and analysts to develop evidence on clinical utility of genomic technologies. Education and training may include legislation, policies, and guidelines for use of genetic information for research, availability and types of electronic data sources and the range of information they contain, and research methods (e.g., observational study designs, strengths and limitations of observational data, methods for linking/merging data, analytical techniques). Researchers should be familiar with and adhere to established methodology standards ${ }^{58}$ that specify a minimum set of recommendations for designing and conducting patient-centered outcomes research including pragmatic trials.

\section{FUNDING AND GOVERNANCE OF THE COLLABORATIVE MODEL}

We propose that our collaborative model could be accomplished as an extension of the US Precision Medicine Initiative. ${ }^{59}$ While the Precision Medicine Initiative is mostly a discovery platform for genetic and environmental causes of human diseases, ${ }^{60}$ our proposed model with its large study size and connectivity of individual patient information with EHR and other sources of data is in line with the initiative. In our model, a portion of the financial burden is shared by payers and manufacturers of tests, and a large component of data infrastructure of existing health networks is already supported by other funding agencies including the FDA and PCORI. A coordinating center is necessary to build the proposed evidence generation program that is important for clinical practice, public health, and health policy development, through subcontracts with collaborating institutions, which may include data and academic partners who provide access to health-care data and scientific, technical, methodologic, and organizational expertise.

For example, Sentinel's coordinating center ${ }^{31}$ oversees active participation by the collaborating institutions and the patient community; leads the development and implementation of data infrastructure, data standards, and quality measures; leads the development and implementation of epidemiologic 
and biostatistics-related analytical approaches; leads decision making relating to priorities of activities; and provides scientific guidance on statistical methods and designs of study protocols. The coordinating center may also consider engaging legal experts and ethicists to provide guidance on privacy and confidentiality of individual health information and related policies. This collaborative model would therefore be codeveloped by private and public sponsors to create a multipurpose and reusable national resource that generates knowledge from data gathered in the course of patient care to drive evidence-based clinical practice and health system changes. The costs for this model are unlikely to be negligible (e.g., $\$ 150$ million $^{61}$ over 5 years for the Sentinel program) but the lack of a system for rapid evidence generation presents substantial opportunity costs because all the investments on genomic science and medicine cannot be fully realized. What we propose is one likely cost-effective approach to evidence generation by leveraging existing resources.

\section{CONCLUSION}

Now is the time to establish a sustainable and structured mechanism for rapid generation of evidence on clinical utility of genomic-based technologies. We believe rapid evidence generation is achievable by risk-sharing agreements between stakeholders, leveraging health-data networks with augmented genomic data to track the use of genomic technologies and monitor clinical outcomes in millions of people, and engaging stakeholders to drive patient-centered research priorities. This collaborative model can create a multipurpose and reusable national resource in which data gathered in the course of routine care are used to generate evidence on clinical utility of promising genomic technologies.

\section{ACKNOWLEDGMENTS}

We are grateful to Caitlin Lupton for administrative assistance.

\section{DISCLOSURE}

G.S.G. has received editor royalties from Elsevier and has served on the advisory boards of Genome, CardioDx, Interleukin Genetics, and Omicia, and the board of directors of Alere. The other authors declare no conflict of interest.

\section{REFERENCES}

1. National Human Genome Research Institute. Regulation of Genetic Tests. 2016. https://www.genome.gov/10002335/regulation-of-genetic-tests/. Accessed 8 June 2017.

2. US Department of Health and Human Services, Food and Drug Administration, Center for Devices and Radiological Health, Office of In Vitro Diagnostics and Radiological Health. Use of Standards in FDA Regulatory Oversight of Next Generation Sequencing (NGS)-Based In Vitro Diagnostics (IVDs) Used for Diagnosing Germline Diseases: Draft Guidance for Stakeholders and Food and Drug Administration Staff. Center for Biologics Evaluation and Research: Rockville, MD, 2016.

3. US Department of Health and Human Service, Food and Drug Administration, Center for Devices and Radiological Health, Office of In Vitro Diagnostics and Radiological Health. Use of Public Human Genetic Variant Databases to Support Clinical Validity for Next Generation Sequencing (NGS)-Based In Vitro Diagnostics: Draft Guidance for Stakeholders and Food and Drug Administration Staff. Center for Biologics Evaluation and Research: Rockville, MD, 2016.
4. Rehm HL, Berg JS, Brooks LD, et al. ClinGen-the Clinical Genome Resource. N Engl J Med 2015;372:2235-2242.

5. Phillips KA, Deverka PA, Sox HC, et al. Making genomic medicine evidence-based and patient-centered: a structured review and landscape analysis of comparative effectiveness research. Genet Med; e-pub ahead of print 13 April 2017.

6. Teutsch SM, Bradley LA, Palomaki GE, et al. The Evaluation of Genomic Applications in Practice and Prevention (EGAPP) Initiative: methods of the EGAPP Working Group. Genet Med 2009;11:3-14.

7. Committee on the Evidence Base for Genetic Testing, Board of Health of Select Populations, Board on Health Care Services, Division HaM. An Evidence Framework for Genetic Testing. National Academies of Sciences: Washington, DC, 2017

8. Grosse SD. Economic analyses of genetic tests in personalized medicine: clinical utility first, then cost utility. Genet Med 2014;16:225-227.

9. Khoury MJ, Berg A, Coates R, Evans J, Teutsch SM, Bradley LA. The evidence dilemma in genomic medicine. Health Aff 2008;27:1600-1611.

10. Khoury MJ. Dealing with the evidence dilemma in genomics and personalized medicine. Clin Pharmacol Ther 2010;87:635-638.

11. Dotson WD, Douglas MP, Kolor K, et al. Prioritizing genomic applications for action by level of evidence: a horizon-scanning method. Clin Pharmacol Ther 2014;95:394-402.

12. Ferrario $A$, Kanavos $P$. Dealing with uncertainty and high prices of new medicines: a comparative analysis of the use of managed entry agreements in Belgium, England, the Netherlands and Sweden. Soc Sci Med 2015;124:39-47.

13. Lu CY. Uncertainties in real-world decisions on medical technologies. Int J Clin Pract 2014;68:936-940.

14. Walker S, Sculpher M, Claxton K, Palmer S. Coverage with evidence development, only in research, risk sharing, or patient access scheme? A framework for coverage decisions. Value Health 2012;15:570-579.

15. Lu CY, Lupton C, Rakowsky S, Babar ZU, Ross-Degnan D, Wagner AK. Patient access schemes in Asia-Pacific markets: current experience and future potential. J Pharm Policy Pract 2015;8:6.

16. Mohr PE, Tunis SR. Access with evidence development: the US experience. Pharmacoeconomics 2010:28:153-162.

17. Centers for Medicare \& Medicaid Services. Pharmacogenomic testing to predict warfarin responsiveness. 2015. https://www.cms.gov/Medicare/ Coverage/Coverage-with-Evidence-Development/Pharmacogenomic-Testingto-Predict-Warfarin-Responsiveness.html. Accessed 1 August 2017.

18. Trueman P, Grainger DL, Downs KE. Coverage with evidence development: applications and issues. Int I Technol Assess Health Care 2010;26:79-85.

19. Academy of Managed Care Pharmacy. BBCIC: Biologics \& Biosimilars, Collective Intelligence Consortium. 2015. http://bbcic.org/. Accessed 8 June 2017.

20. Lu CY, Cohen JP. Can genomic medicine improve financial sustainability of health systems? Mol Diagn Ther 2015;19:71-77.

21. Ginsburg G. Medical genomics: gather and use genetic data in health care. Nature 2014;508:451-453.

22. Chambers DA, Feero WG, Khoury MJ. Convergence of implementation science, precision medicine, and the learning health care system: a new model for biomedical research. JAMA 2016;315:1941-1942.

23. Institute of Medicine (US) Roundtable on Value \& Science-Driven Health Care. Clinical Data as the Basic Staple of Health Learning: Creating and Protecting a Public Good: Workshop Summary. US National Academies Press: Washington, DC, 2010.

24. Avorn J, Kesselheim AS. The 21st Century Cures Act-will it take us back in time? N Engl J Med 2015;372:2473-2475.

25. Kimmel SE, French $B$, Kasner SE, et al. A pharmacogenetic versus a clinical algorithm for warfarin dosing. N Engl J Med 2013;369: 2283-2293.

26. Dolor RJ, Schmit KM, Graham DG, Fox CH, Baldwin LM. Guidance for researchers developing and conducting clinical trials in practicebased research networks (PBRNs). J Am Board Fam Med 2014;27: 750-758.

27. Thompson P, Wheeler HE, Delaney SM, et al. Pharmacokinetics and pharmacogenomics of daunorubicin in children: a report from the Children's Oncology Group. Cancer Chemother Pharmacol 2014;74: 831-838.

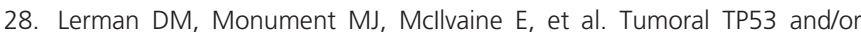
CDKN2A alterations are not reliable prognostic biomarkers in patients with localized Ewing sarcoma: a report from the Children's Oncology Group. Pediatr Blood Cancer 2015;62:759-765. 
29. Habel LA, Shak S, Jacobs MK, et al. A population-based study of tumor gene expression and risk of breast cancer death among lymph nodenegative patients. Breast Cancer Res 2006;8:R25.

30. Gottesman O, Kuivaniemi H, Tromp G, et al. The Electronic Medical Records and Genomics (eMERGE) Network: past, present, and future. Genet Med 2013;15:761-771.

31. Curtis LH, Brown J, Platt R. Four health data networks illustrate the potential for a shared national multipurpose big-data network. Health Aff 2014;33:1178-1186.

32. Toh S, Baker MA, Brown JS, Kornegay C, Platt R. Rapid assessment of cardiovascular risk among users of smoking cessation drugs within the US Food and Drug Administration's Mini-Sentinel program. JAMA Intern Med 2013;173:817-819.

33. Toh S, Reichman ME, Houstoun $M$, et al. Comparative risk for angioedema associated with the use of drugs that target the reninangiotensin-aldosterone system. Arch Intern Med 2012;172:1582-1589.

34. Fleurence RL, Curtis LH, Califf RM, Platt R, Selby JV, Brown JS. Launching PCORnet, a national patient-centered clinical research network. J Am Med Inform Assoc 2014;21:578-582.

35. Hoffman MA, Williams MS. Electronic medical records and personalized medicine. Hum Genet 2011;130:33-39.

36. Starren J, Williams MS, Bottinger EP. Crossing the omic chasm: a time for omic ancillary systems. JAMA 2013;309:1237-1238.

37. Epstein RS, Moyer TP, Aubert RE, et al. Warfarin genotyping reduces hospitalization rates results from the MM-WES (Medco-Mayo Warfarin Effectiveness study). J Am Coll Cardiol 2010;55:2804-2812.

38. IGNITE: Implementing GeNomics In pracTicE. 2017. https://ignitegenomics.org/. Accessed 19 April 2017.

39. Toh S, Reichman ME, Houstoun M, et al. Multivariable confounding adjustment in distributed data networks without sharing of patientlevel data. Pharmacoepidemiol Drug Saf 2013;22:1171-1177.

40. Toh S, Shetterly S, Powers JD, Arterburn D. Privacy-preserving analytic methods for multisite comparative effectiveness and patient-centered outcomes research. Med Care 2014;52:664-668.

41. Toh S, Gagne JJ, Rassen JA, Fireman BH, Kulldorff M, Brown JS. Confounding adjustment in comparative effectiveness research conducted within distributed research networks. Med Care 2013;51(8 Suppl 3):S4-10.

42. Lu CY, Penfold R, Toh S, et al. Near real-time surveillance for consequences of health policies using sequential analysis. Med Care (in press).

43. Khoury MJ, Ioannidis JP. Medicine. Big data meets public health. Science 2014;346:1054-1055.

44. Weitzel KW, Alexander M, Bernhardt BA, et al. The IGNITE network: a model for genomic medicine implementation and research. BMC Med Genomics 2016;9:1.

45. PatientsLikeMe. Live better, together! 2016. https://www. patientslikeme. com/. Accessed 8 June 2017.

46. ClinGen: Clinical Genome Resource. GenomeConnect. 2016. https:// www.clinicalgenome.org/genomeconnect/. Accessed 8 June 2017.
47. SFARI: Simons Foundation Autism Research Initiative. Simons Foundation. 2016. https://sfari.org/. Accessed 8 June 2017.

48. Parent Project Muscular Dystrophy. Parent Project Muscular Dystrophy: Leading the Fight to End Duchenne. 2016. http://www.parentprojectmd. org/site/PageServer?pagename=nws_index. Accessed 8 June 2017.

49. Pear R. Uncle Sam wants you-or at least your genetic and lifestyle information. New York Times. 31 October 2016.

50. Issa AM, Tufail W, Hutchinson J, Tenorio J, Baliga MP. Assessing patient readiness for the clinical adoption of personalized medicine. Public Health Genomics 2009;12:163-169.

51. Haga SB, Kawamoto K, Agans R, Ginsburg GS. Consideration of patient preferences and challenges in storage and access of pharmacogenetic test results. Genet Med 2011;13:887-890.

52. Haga SB, O’Daniel JM, Tindall GM, Lipkus IR, Agans R. Public attitudes toward ancillary information revealed by pharmacogenetic testing under limited information conditions. Genet Med 2011;13:723-728.

53. Haga SB, O'Daniel JM, Tindall GM, Lipkus IR, Agans R. Survey of US public attitudes toward pharmacogenetic testing. Pharmacogenomics J 2012;12:197-204.

54. Carey DJ, Fetterolf SN, Davis FD, et al. The Geisinger MyCode community health initiative: an electronic health record-linked biobank for precision medicine research. Genet Med 2016;18:906-913.

55. The White House. Precision Medicine Initiative: Proposed Privacy and Trust Principles. Available from: https://obamawhitehouse.archives.gov/ sites/default/files/microsites/finalpmiprivacyandtrustprinciples.pdf.

56. Green RC, Lautenbach D, McGuire AL. GINA, genetic discrimination, and genomic medicine. N Engl J Med 2015;372:397-399.

57. Snyder SR, Mitropoulou C, Patrinos GP, Williams MS. Economic evaluation of pharmacogenomics: a value-based approach to pragmatic decision making in the face of complexity. Public Health Genomics 2014; 17:256-264.

58. PCORI: Patient-Centered Outcomes Research Institute. 9. Standards for adaptive and Bayesian trial designs. 2013. http://www.pcori.org/ research-results/research-methodology/pcori-methodology-standards\# Adaptive\%20and\%20Bayesian\%20Trial\%20Designs. Accessed 8 June 2017.

59. The White House. The Precision Medicine Initiative. 2016. https://www. whitehouse.gov/precision-medicine. Accessed 8 June 2017.

60. Precision Medicine Initiative (PMI) Working Group. The Precision Medicine Initiative Cohort Program-Building a Research Foundation for 21st Century Medicine 2015. https://www.nih.gov/sites/default/files/ research-training/initiatives/pmi/pmi-working-group-report-20150917-2. pdf.

61. Findlay S. The FDA's Sentinel Initiative. The Food and Drug Administration has launched a new system to assess the safety of prescription drugs after they are approved for sale. Health Affairs and Robert Wood Johnson Foundation; 2015. http://healthaffairs.org/healthpolicybriefs/brief_pdfs/ healthpolicybrief_139.pdf. 